Article

\title{
Hybrid Genetic Algorithm Fuzzy-Based Control Schemes for Small Power System with High-Penetration Wind Farms
}

\author{
Mohammed Elsayed Lotfy ${ }^{1,2, *}$ (1) , Tomonobu Senjyu ${ }^{2}$, Mohammed Abdel-Fattah Farahat ${ }^{1}$, \\ Amal Farouq Abdel-Gawad ${ }^{1}$, Liu Lei $^{2}$ and Manoj Datta ${ }^{3}$ \\ 1 Department of Electrical Power and Machines, Zagazig University, Zagazig 44519, Egypt; \\ ma_farahat@zu.edu.eg (M.A.-F.F.); amgawad2001@yahoo.com (A.F.A.-G.) \\ 2 Department of Electrical and Electronics Engineering, University of the Ryukyus, Okinawa 903-0213, Japan; \\ b985542@tec.u-ryukyu.ac.jp (T.S.); LL381456401@yahoo.co.jp (L.L.) \\ 3 Department of Electrical and Biomedical Engineering, RMIT University, Melbourne, Victoria 3001, Australia; \\ manoj.datta@rmit.edu.au \\ * Correspondence: mohamedabozed@zu.edu.eg, Tel.: +81-98-895-8686
}

Received: 2 February 2018; Accepted: 1 March 2018; Published: 4 March 2018

Simple Summary: This paper can be considered as a complete study clarifying the impact of implementing high-penetration renewable energy sources in small power systems from the frequency and active power points of view

\begin{abstract}
Wind is a clean, abundant, and inexhaustible source of energy. However, wind power is not constant, as windmill output is proportional to the cube of wind speed. As a result, the generated power of wind turbine generators (WTGs) fluctuates significantly. Power fluctuation leads to frequency deviation and voltage flicker inside the system. This paper presents a new methodology for controlling system frequency and power. Two decentralized fuzzy logic-based control schemes with a high-penetration non-storage wind-diesel system are studied. First, one is implemented in the governor of conventional generators to damp frequency oscillation, while the other is applied to control the pitch angle system of wind turbines to smooth wind output power fluctuations and enhance the power system performance. A genetic algorithm (GA) is employed to tune and optimize the membership function parameters of the fuzzy logic controllers to obtain optimal performance. The effectiveness of the suggested controllers is validated by time domain simulation for the standard IEEE nine-bus three-generator test system, including three wind farms. The robustness of the power system is checked under normal and faulty operating conditions.
\end{abstract}

Keywords: fuzzy control; frequency control; genetic algorithm; pitch angle control; power system stability; wind power generation

\section{Introduction}

Global warming is one of the most serious environmental problems facing the world today. Most remote areas and isolated communities depend on diesel generators to meet electricity demands [1]. Diesel fuel has several drawbacks, including the fact that it is expensive because transportation to remote areas adds extra cost. Moreover, it emits sulfur oxide and carbon dioxide by engine exhaust that is harmful to the environment [1,2]. Due to the environmental and economic effects of the diesel generator, interest in alternative, cost-effective, and clean energy sources has grown significantly. Wind turbines are attracting a lot of attention, and represent the fastest growing and most widely utilized form of renewable energy technology in power systems [3]. Wind-diesel hybrid power systems can be 
installed in isolated communities to improve quality of life. Many small scale wind farms are connected to the distribution network, while many wind farms of $50 \mathrm{MW}$ or more are directly connected to the transmission network [4]. This results in many challenges. Among them is the fact that wind power is not constant and can diverge quickly, since it is proportional to the cube of wind speed. Wind power fluctuations lead to some problems, which can be summarized as follows $[4,5]$ :

1. Wind power fluctuations may cause the grid frequency to fluctuate.

2. The variation in wind speed causes variations in the active power generated and thus the absorbed reactive power by the induction generator from the grid, which leads to voltage flicker at the buses of the power grid.

3. Poor power quality and instability problems appear in the power system as a result of frequency fluctuation and voltage flicker, especially in the case of sensitive loads for frequency and high-voltage deviations. The effects of these problems appear clearer in isolated power systems as penetration of the wind turbine generator (WTG) increases.

Therefore, various power smoothing methods are applied to wind energy conversion systems (WECSs) to overcome these problems. Many pitch angle control techniques are utilized to smooth the output power of WTGs using model predictive controls (MPCs) [6], the genetic algorithm (GA) [7], linear quadratic Gaussian (LQG) [8,9] (which provide robustness in terms of phase and gain margins), and the GA with particle swarm optimization (PSO) [10]. However, the performance of all these linear controllers is limited due to the highly nonlinear characteristics of wind turbines. Fuzzy logic has been applied for pitch angle control [11-14]. However, these studies differ from each other significantly by type and number of inputs and outputs, number and type of input and output membership functions, type of control rules, inference engines, and defuzzification method. No complete studies of the power system performance from the voltage and frequency stability point of view nor evaluations of the robustness of the proposed controllers under severe operating conditions such as generator outage or transmission line faults have been presented. The $H_{\infty}$ control method is used to smooth the output power of WTGs [15-17]. However, the weighting functions in the $H_{\infty}$ control design cannot be easily selected, and this affects the process of design significantly. Also, the order of the $H_{\infty}$ controller depends on that of the plant. This causes a complex structure which is not easy to realize in practical applications, especially for large-scale systems. On the other hand, the frequency control approach in power systems has a long history and there is plenty of related literature. Power system frequency can be negatively affected due to wind power fluctuations, especially with high-penetration wind farms. This leads to a frequency deviation from its nominal value. As a result, under/over frequency relays may operate and disconnect some portions of system loads and generation. However, there are only a few articles on the frequency regulation schemes in the presence of wind turbines. The impact of wind power generation on system frequency response has been discussed using $H_{\infty}$ and $\mu$-synthesis [18], MPC [19], fuzzy logic control (FLC) with the quasi-oppositional harmony search (QOHS) algorithm for the PID controller [20], $\varepsilon$-MOGA [21,22], and the polar fuzzy [23] approaches. These studies use simplified linear models neglecting the nonlinear specifications of wind turbines. Hence, they are very difficult to apply to large-scale power systems with nonlinearities. On the other hand, FLC with PSO [24], FLC with PSO for the PI controller [25], and the PI controller alone [26] have been studied for frequency regulation in the presence of wind turbines without considering the power and voltage stability of the power system. Recently, there have been studies on adaptive FLC approaches to multi-input multi-output (MIMO) nonlinear systems with time-varying delays and input saturation [27], unknown control directions and unknown dead-zones [28], single-input single-output (SISO) with input saturation [29], and packet dropouts and parameter uncertainties using the type-2 fuzzy model-based technique. However, the main drawback for these approaches is the fact that the design parameters [27-29] and the weighting functions [30] selection process affect the system performance significantly. Hence, the parameters should be adjusted carefully for achieving suitable transient performance and control action in practical applications. In [31], an 
adaptive fuzzy backstepping control of a nonlinear system with sampled and delayed measurements was presented. The major disadvantage of this scheme that it suffers from the problem of explosion of complexity, which is caused by the repeating differentiations of nonlinear functions. As a result, the complexity of the controller grows sharply as the order of the system increases, which makes it difficult for practical implementation, especially for large-scale systems. A complete review for hybrid system configuration, modeling, renewable energy sources, criteria for hybrid system optimization and control strategies, and software used for optimal sizing was presented in [32]. A real case study of comparative various standalone hybrid combinations for a remote area in Barwani, India was analyzed according to total net cost and emissions. Dynamic operation and control strategies for a microgrid hybrid wind-photovoltaic fuel cell power system were examined in [33]. In addition, a static var compensator (SVC) was utilized to supply reactive power and regulate the voltage of the hybrid system. Moreover, a general regression neural network (GRNN) with an improved PSO was applied to analyze the performance of the system. Furthermore, a high-performance online training radial basis function network sliding mode (RBFNSS) was designed to drive the optimal wind turbine speed for the maximum power point tracking (MPPT) control scheme. Two MPPT control approaches for both photovoltaic power and the WTG were presented in [34]. The pitch angle control of the WTG used the improved Eleman neural network (ENN) controller and the output was fed to wind turbine to achieve MPPT. In addition, the solar array was integrated with radial basis function network (RBFN) control algorithm to track maximum power. A design of fuzzy logic-based voltage-regulated solar power MPPT system was introduced in [35]. The voltage-regulated MPPT scheme was formulated as a feedback control system where the maximum power point condition and voltage regulation requirements were used as control systems input. Moreover, a buck-boost converter-based fuzzy controller was designed for the voltage-regulated MPPT system considering the error of the maximum power point condition and the error of the voltage regulation as two input variables for the fuzzy controller. A control approach to enhance the phase correction adjustment of distribution transformers in a grid was discussed in [36] using hybrid bird mating optimization (HBMO). The proposed technique was performed through open wye-open delta transformers under three practical systems and compared to those approaches that use other optimal techniques such as the GA, PSO, and conventional bird mating optimization (BMO). The outcomes of this research showed the ability of the proposed scheme to improve the voltage imbalance and deviation. A novel unsymmetrical faults analysis technique based on the actual three-phase models and boundary conditions was analyzed in [37]. Two relationship matrices presented by the bus injection to branch the current matrix and the branch current to bus voltage mismatch matrix were used to achieve the power flow solutions. These two matrices were used to observe the relation between bus voltage and bus current injections. The presented scheme can be used to solve assorted types of single or simultaneous unsymmetrical faults. A direct building algorithm for microgrid distribution ground fault analysis was discussed in [38]. Four possibilities of network topology changes were considered for ground fault analysis. In addition, research analyzed the ground fault model of a battery as a distributed energy resource which can be used for both islanded and grid-connected modes. An optimal sizing approach of hybrid grid-connected photovoltaic wind power systems for real hourly wind and solar irradiation data and electricity demand from a certain location was presented in [39]. The methodology was tested by means of a case study in which the actual hourly electricity retail and market prices were implemented to obtain a realistic estimation of life cycle costs and benefits. A sensitivity analysis that allows the detection of the variables for which the system is more sensitive was also performed. A novel intelligent damping controller (NIDC) for the static synchronous compensator (STATCOM) was discussed in [40] to damp power system oscillations. The proposed NIDC consists of a PID linear controller and functional link-based novel recurrent fuzzy neural network (FLNRFNN). The NIDC for the STATCOM was developed to improve both transient stability and system oscillations. The NIDC is unlike conventional controllers, the performance of which degrades for such changes and retuning is required to produce the desired performance. A new cascaded PD-fuzzy PID controller 
was analyzed in [41] for a hybrid source unified power system for automatic generation control. The optimization of scaling parameters of the suggested controller was performed using the hybrid grey wolf optimization teaching-learning-based optimization (GWO-TLBO) technique. Both the AC tie line and AC-DC tie line were considered when analyzing the dynamic performance of the system. Furthermore, the frequency stability of the system was improved by employing unified power flow controller (UPFC) in the system. Moreover, various architectures, design criteria, sizing methodologies, and a control paradigm of an integrated renewable hybrid energy system were presented in [42]. A practical hybrid energy system depending on various parameters such as technological, economical, socio-political, and environmental factors was considered.

For all these reasons, this paper proposes a new control approach in a small power system consisting of the standard IEEE nine-bus three-generator test model with three high-penetration wind farms. The proposed method is accessible by implementing two decentralized FLC-based schemes. First, one is applied for the governor of conventional generators to mitigate frequency deviations. Meanwhile, the latter is constructed in the pitch angle system of wind turbines to smooth the wind output power fluctuations and therefore enhance the total system performance. The GA is used to tune the input and output membership function parameters of the fuzzy logic controllers to ensure the best performance. The effectiveness of the proposed technique is verified by the numerical simulations in the MATLAB ${ }^{\circledR} /$ SIMULINK $^{\circledR}$ environment under normal and faulty operating conditions. This research is considered as a continuation and modification of authors' previous studies [21-23] that used simplified linear models neglecting the high nonlinear behavior of wind turbine. Moreover, in the authors' previous work, FLC without any optimization technique was used, which made the mission of deciding the input and output membership functions parameters complex and time-consuming, which may not often lead to satisfying results. The main contributions of the proposed control approach can be summarized as follows:

1. The proposed control scheme presents two fuzzy logic-based controllers to treat the drawbacks of the other linear control approaches that neglect the highly nonlinear characteristics of wind turbines.

2. The intended technique uses the GA to tune the membership function parameters of FLC to achieve optimal performance and at the same time to eliminate the main disadvantage of FLC presented in the fact that deciding the input and output membership parameters of FLC is a time-consuming and complex task which often may not lead to satisfactory results.

3. The proposed GA-FLC scheme discusses a complete study for the power system performance from the power and frequency stability point of views and their vital effects to improve the total behavior of system. This is distinct from the majority of the previous works which concentrate on one type of stability for the power system.

4. The presented control approach is tested under severe operating conditions such as a three-phase to ground fault at the most loaded transmission line to confirm its effectiveness and robustness and check its performance during the fault period to prove its capability to keep frequency and power within its acceptable limits, so that under/over frequency or power relays do not disconnect any portion of the power system. Testing was also performed to investigate that the system can safely return to normal operation after fault clearance. Hence, using this hybrid control scheme in practical applications can ensure a continuous operation of the power system with high power quality provided even under emergency conditions.

This paper is organized as follows: Section 2 describes the small power system configuration. Section 3 discusses the proposed GA-fuzzy based control design approaches including a brief introduction for FLC and GA. Section 4 presents the simulation results for four case studies of the small power system. Section 5 analyzes the time domain simulated results of the four studied cases of the power system. Specific conclusions are then drawn in Section 6. 


\section{System Configuration}

The standard IEEE three-generator nine-bus system is considered as the test model in this study to inspect the performance of the proposed control schemes. The single line diagram of the test system is presented in Figure 1. This model consists of 3 generators, 3 loads, 6 transmission lines and 3 transformers whose simulation parameters are indicated in [43]. The model is updated by adding three wind farms. The total system installed capacity is $567.5 \mathrm{MW}$ of conventional generation and $189 \mathrm{MW}$ of average wind power generation ( $25 \%$ penetration). Each wind farm is composed of 21 wind turbines and every turbine has a 3-MW rating. The fixed speed squirrel cage induction generator is used in this study to investigate its achievement under various operating conditions with/without the proposed control approaches.

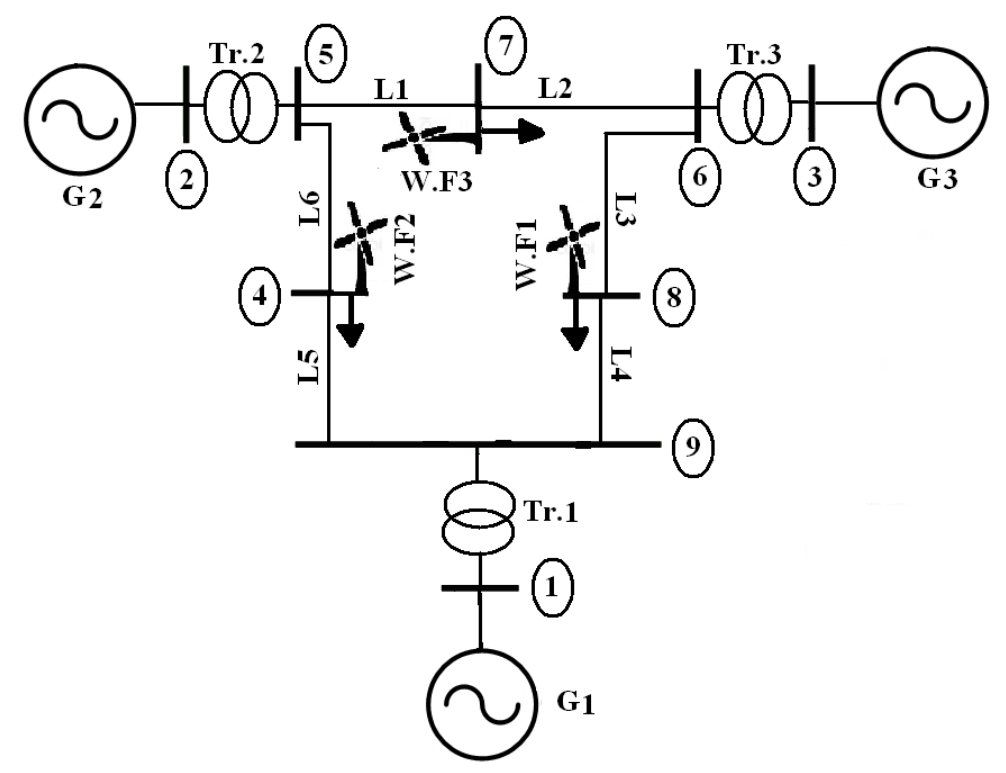

Figure 1. Single-line diagram of the nine-bus system.

\section{Controller Design Approach}

In this study, two FLC schemes have been proposed to improve the performance of a small power system with high-penetration wind farms. The GA is used to tune the input and output membership function parameters of the two fuzzy logic controllers to enhance their overall achievement.

\subsection{Fuzzy Logic Control}

The FLC technique becomes a good replacement for the conventional control methods. It can perform efficiently when the operating conditions change rapidly. Hence, these features make it very effective for power system applications, especially with uncertain, incomplete, and non-linear data from wind turbine systems [44,45]. The FLC consists of three steps: fuzzification, knowledge base creation, and defuzzification.

1. Fuzzification is the process of transforming the crisp input signals into fuzzy set variables as FLC cannot handle these input signals.

2. The knowledge base is the heart of fuzzy system, as it determines how fuzzy logic operations are performed. It consists of fuzzy IF-THEN rules. The database contains the membership functions of fuzzy subsets. A fuzzy rule includes fuzzy variables and the fuzzy subset described by membership function. Triangular, trapezoid, and Gaussian functions are the more standard membership functions used in FLC. 
3. Defuzzification is used to convert the fuzzy output to a crisp value using membership functions like the ones utilized in the process of fuzzification. There are some common defuzzification methods such as center of sums, mean- max, weighted average, and center of gravity [46,47].

\subsection{Genetic Algorithm}

The GA is an optimization method that based on the mechanism of natural selection and natural genetics. The search process is very similar to the natural evolution of biological creatures in which successive generations of organisms are born and raised until they are able to breed. Just like in the animal kingdom, only the fittest will survive to produce, while the weakest will be omitted [48]. The GA can be applied to solve a variety of optimization problems that are not well suited for standard optimization algorithms such as problems in which the objective function is discontinuous, non-differentiable, stochastic, or highly nonlinear [49]. The algorithm begins by creating a random initial population. Then, it uses the current population to create the children to make up the next generation. The algorithm selects a group of individuals in the current population, called parents, who contribute their genes to their children. It usually selects individuals that have better fitness values as parents. The GA creates three types of children for the next generation [50]:

1. Elite children are individuals in the current generation with the best fitness values. These individuals automatically survive to the next generation.

2. Crossover children are created by combining the vectors of a pair of parents.

3. Mutated children are created by introducing random changes to a single parent. Both processes of crossover and mutation are essential to the GA. Crossover enables the algorithm to extract the best genes from different individuals and recombine them to get potentially superior children. While, mutation improves the diversity of the population to increase the probability that the algorithm will generate individuals with better fitness values. The algorithm is repeated for many generations till stops if one of the stopping criteria is met.

\subsection{Proposed GA-Fuzzy-Based Control Schemes}

\subsubsection{Governor Control System}

The basic construction of the governor control approach designed for the conventional generators is presented in Figure 2. The deviation of rotor speed of each conventional generator and its variation during a sampled time are considered as two input signals for the fuzzy controller, while its output is the control signal to adjust the governor set point of each generator. The governor has non-linear characteristics, but can be modeled with a first-order lag transfer function where the time constant is $0.08 \mathrm{~s}$. Also, the value of $R$ is $2.4 \mathrm{~Hz} /$ pu.Mw. Fuzzy membership functions for the input and output patterns of the proposed FLC technique are addressed in Figure 3. Also, Table 1 shows the associated control rules. The membership functions corresponding to the input and output variables are set as Negative Large (NL), Negative Medium2 (NM2), Negative Medium1 (NM1), Negative Small (NS), Zero (Z), Positive Small (PS), Positive Medium1 (PM1), Positive Medium2 (PM2), and Positive Large (PL). They have been arranged based on triangular membership functions. The antecedent part of each IF-THEN rule is created using the And function. Also, Mamdani fuzzy interference system is used in this control approach. Finally, resultant fuzzy subsets representing the controller output are converted to the crisp values. Unlike the traditional control theorems, which are based on the linearized models of the controlled system, the fuzzy control scheme tries to build the proposed controller in a direct way based on long-term experience, knowledge of domain operators, and measurements. Then, the GA is used to tune the input and output membership function parameters of the proposed fuzzy controller to 
improve its performance using integral absolute error criteria (IAE) utilizing the MATLAB ${ }^{\circledR}$ (Release 2016a, The MathWorks, Inc., Natick, MA, USA) environment based on the following equation:

$$
\text { Fitness Function }=\int_{0}^{t}|\Delta f| d t .
$$

The optimal values for membership function parameters obtained from the GA are then listed in Table 2. In Table 2, unit of the input membership functions parameters $\left(A_{1}-A_{14}\right)$ is $\mathrm{Hz}$ while the unit of the output membership functions parameters $\left(A_{15}-A_{21}\right)$ is pu.Mw.

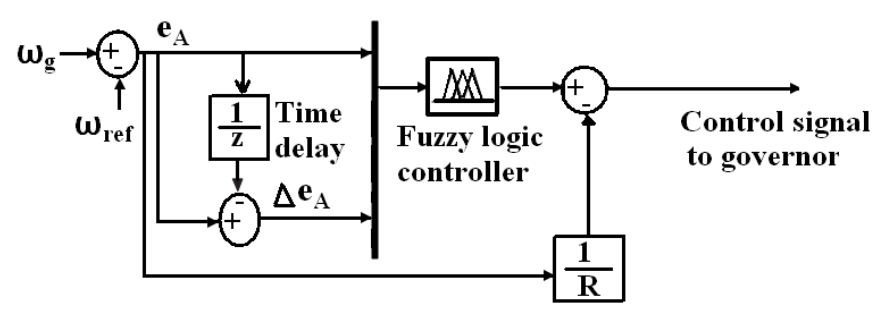

Figure 2. Governor control scheme.
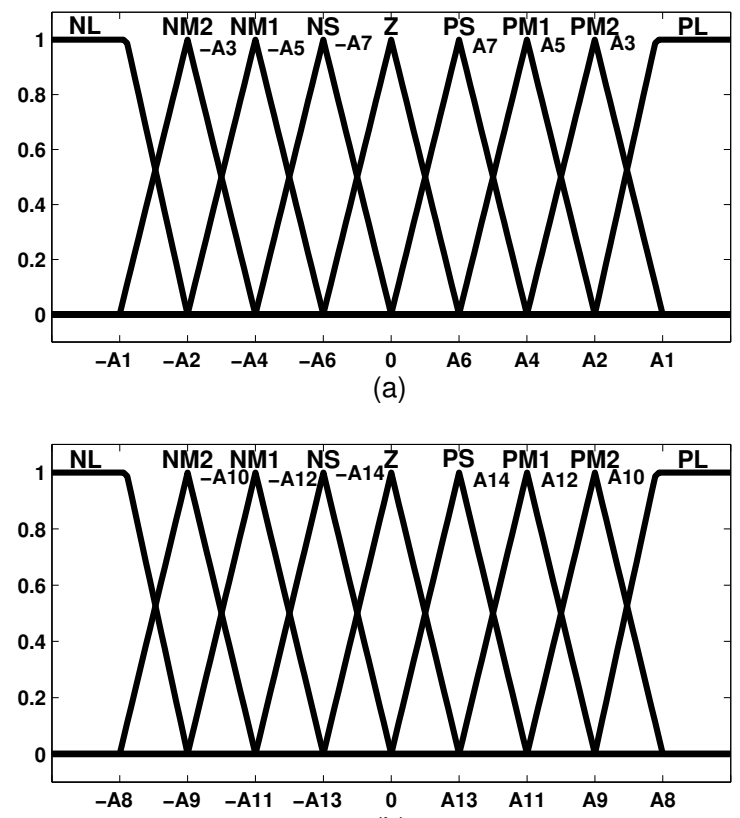

(b)

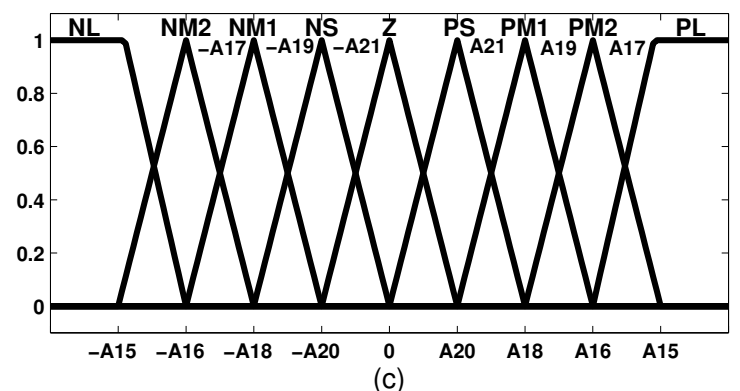

Figure 3. (a) Fuzzy membership functions for the governor controller's first input; (b) Fuzzy membership functions for the governor controller's second input; (c) Fuzzy membership functions for the governor controller's output. NL: Negative Large; NM2: Negative Medium2; NM1: Negative Medium1; NS: Negative Small; Z: Zero; PS: Positive Small; PM1: Positive Medium1; PM2: Positive Medium2; PL: Positive Large. 
Table 1. Fuzzy control rules.

\begin{tabular}{|c|c|c|c|c|c|c|c|c|c|}
\hline$\Delta e_{A}$ & NL & NM2 & NM1 & NS & Z & PS & PM1 & PM2 & PL \\
\hline NL & PL & PL & PM2 & PM2 & PM1 & PM1 & PS & PS & Z \\
\hline NM2 & PL & PM2 & PM2 & PM1 & PM1 & PS & $\mathrm{Z}$ & NS & NS \\
\hline NM1 & PL & PM2 & PM1 & PM1 & PS & Z & Z & NS & NM1 \\
\hline NS & PL & PM2 & PM1 & PS & PS & $\mathrm{Z}$ & NS & NS & NM1 \\
\hline Z & PM2 & PM1 & PS & PS & Z & NS & NM1 & NM1 & NM2 \\
\hline PS & PM1 & PS & PS & Z & NS & NM1 & NM1 & NM2 & NL \\
\hline PM1 & PS & PS & Z & $\mathrm{Z}$ & NS & NM1 & NM2 & NM2 & NL \\
\hline PM2 & PS & $\mathrm{Z}$ & Z & NS & NM1 & NM2 & NM2 & NL & NL \\
\hline PL & Z & NS & NM1 & NM2 & NM2 & NL & NL & NL & NL \\
\hline
\end{tabular}

Table 2. Optimal membership function parameter values.

\begin{tabular}{llllllllllll}
\hline Variable & A1 & A2 & A3 & A4 & A5 & A6 & A7 & A8 & A9 & A10 & A11 \\
\hline Value & 0.078 & 0.058 & 0.066 & 0.05 & 0.031 & 0.017 & 0.013 & 0.082 & 0.072 & 0.076 & 0.039 \\
Variable & A12 & A13 & A14 & A15 & A16 & A17 & A18 & A19 & A20 & A21 & \\
Value & 0.061 & 0.017 & 0.035 & 0.541 & 0.331 & 0.482 & 0.253 & 0.291 & 0.157 & 0.223 & \\
\hline
\end{tabular}

\subsubsection{Pitch Angle Control System}

The basic configuration of the WTG system is presented in Figure 4. Figure 5 shows the structure of the proposed pitch angle control scheme that determines the $\beta$ of each wind turbine. The fraction of extracted wind power converted to mechanical power of the rotor, $P_{w}$, can be calculated as follows:

$$
P_{w}=\frac{1}{2} \times C_{p} \times V_{w}^{3} \times \rho \times A
$$

where $\mathrm{A}=\pi r^{2}$. Values of $r$ and $\rho$ are $23.5 \mathrm{~m}$ and $1.25 \mathrm{Kg} / \mathrm{m}^{3}$, respectively. $C_{p}$ is a function of both $\lambda$ and $\beta$. $\lambda$ is defined as the ratio of the speed at the blade tip to the wind speed and can be expressed as:

$$
\lambda=\frac{r \times \omega_{w}}{V_{w}}
$$

Then, an approximate expression for $C_{p}$ as function of $\lambda$ and $\beta$ can be given as [51]:

$$
C_{p}=(0.44-0.0167 \beta) \sin \left(\frac{\pi(\lambda-3)}{15-0.3 \beta}\right)-0.0184(\lambda-3) \beta
$$

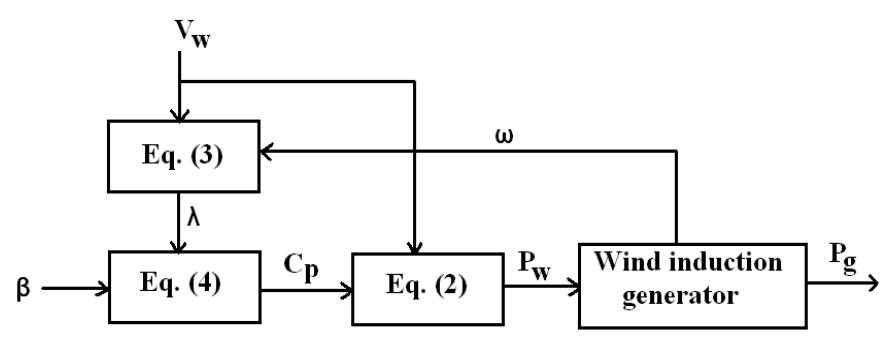

Figure 4. System configuration of the wind turbine generator (WTG). 


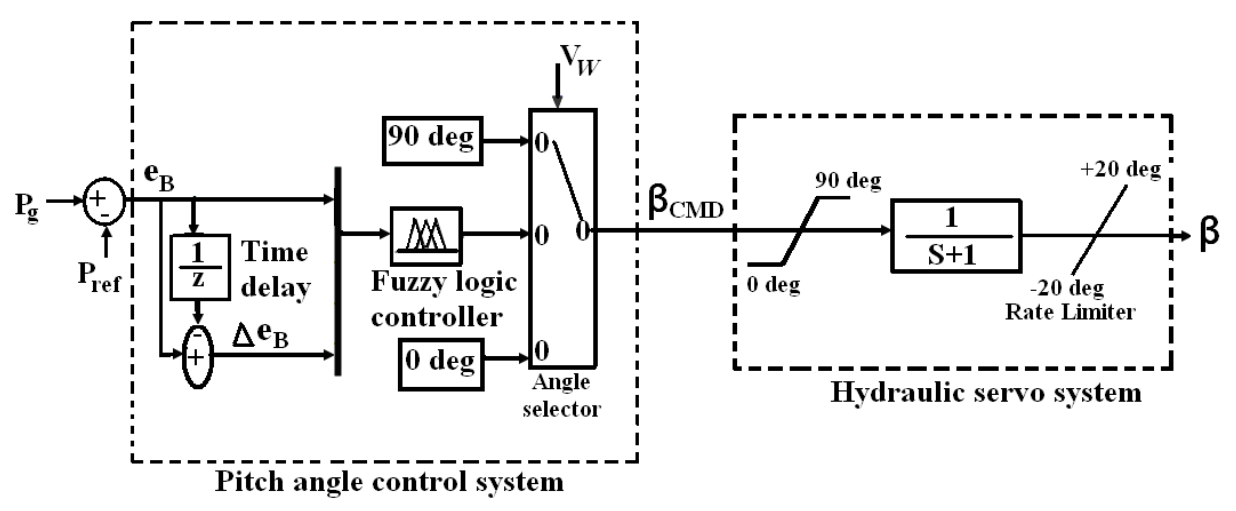

Figure 5. Pitch angle control system structure.

The hydraulic servo system has nonlinear characteristics. However, it can be modeled as first-order lag system. Therefore, in this control scheme, the first-order lag system is considered where the time constant is $1 \mathrm{~s}$. Fuzzy sets and their corresponding membership functions for the proposed controller are presented in Figure 6, while their control rules are discussed in Table 3. The deviation of output power for each WTG and its change with time is then used as input signals for the fuzzy controller, while output is the control signal to the hydraulic servo system that drives the blades according to $\beta_{C M D}$. The membership functions of the first input are set as Negative (N), Zero (Z), Small (S), Medium1 (M1), Medium2 (M2), and Large (L). Also, the membership functions corresponding to the second input are held as Negative Large (NL), Negative Medium (NM), Negative Small (NS), Zero (Z), Positive Small (PS), Positive Medium (PM), and Positive Large (PL). Meanwhile, the output membership functions are arranged as Zero $(\mathrm{Z}), \mathrm{xSmall}(\mathrm{xS})$, Small $(\mathrm{S}), \mathrm{xMedium}(\mathrm{xM})$, xxMedium $(\mathrm{xxM})$, xxxMedium (xxxM), Large (L), xLarge ( $x \mathrm{~L})$, and xxLarge $(x x L)$. The triangular membership function which is the most popular is also used in this control approach. The output of each rule is composed by using the And function with interpretation of the minimum. In the same manner as the first control scheme, the Mamdani interference system is used here. For the sake of applying GA in the intended pitch angle control approach to enhance its performance, the MATLAB ${ }^{\circledR}$ (Release 2016a, The MathWorks, Inc., Natick, MA, USA) environment is utilized with the following fitness function:

$$
\text { Fitness Function }=\int_{0}^{t}\left|\Delta P_{W} t g\right| d t .
$$

Following the usage of the GA, the optimal values for membership functions parameters acquired are then scored in Table 4. In Table 4, the unit of the input membership functions parameters $\left(B_{1}-B_{12}\right)$ is pu.Mw. Meanwhile, the units of the output membership functions parameters $\left(B_{13}-B_{27}\right)$ are in degrees. The flowchart of the complete design process for the proposed two GA-fuzzy based control schemes is presented in Figure 7.

Table 3. Control rules of a pitch angle controller. N: Negative; Z: Zero; S: Small; M1: Medium1; M2: Medium2; L: Large; NL: Negative Large; NM: Negative Medium; NS: Negative Small; S: Small (S); L: Large.

\begin{tabular}{|c|c|c|c|c|c|c|c|}
\hline$e_{B}$ & NL & NM & NS & Z & PS & PM & PL \\
\hline $\mathrm{N}$ & Z & Z & Z & Z & Z & Z & Z \\
\hline Z & Z & Z & Z & Z & Z & Z & Z \\
\hline$S$ & Z & XS & XS & XS & $S$ & $S$ & $\mathrm{XM}$ \\
\hline M1 & XS & S & S & $\mathrm{XM}$ & XM & $\mathrm{XM}$ & XXM \\
\hline M2 & S & S & $\mathrm{XM}$ & XM & XXM & XXM & $\mathrm{XXXM}$ \\
\hline $\mathrm{L}$ & $\mathrm{XM}$ & XXM & XXM & XXXM & L & XL & XXL \\
\hline
\end{tabular}


Table 4. Optimal membership function parameter values.

\begin{tabular}{llllllllll}
\hline Variable & B1 & B2 & B3 & B4 & B5 & B6 & B7 & B8 & B9 \\
\hline Value & 0.0342 & 0.0083 & 0.0453 & 0.0388 & 0.082 & 0.083 & 0.115 & 0.0125 & 0.012 \\
Variable & $\mathrm{B} 10$ & $\mathrm{~B} 11$ & $\mathrm{~B} 12$ & $\mathrm{~B} 13$ & $\mathrm{~B} 14$ & $\mathrm{~B} 15$ & $\mathrm{~B} 16$ & $\mathrm{~B} 17$ & $\mathrm{~B} 18$ \\
Value & 0.00122 & 0.0021 & 0.00432 & 2.43 & 3.87 & 8.56 & 6.24 & 13.86 & 12.13 \\
Variable & $\mathrm{B} 19$ & $\mathrm{~B} 20$ & $\mathrm{~B} 21$ & $\mathrm{~B} 22$ & $\mathrm{~B} 23$ & $\mathrm{~B} 24$ & $\mathrm{~B} 25$ & $\mathrm{~B} 26$ & $\mathrm{~B} 27$ \\
Value & 18.46 & 16.45 & 23.93 & 22.72 & 36.84 & 34.13 & 52.62 & 58.32 & 59.26 \\
\hline
\end{tabular}
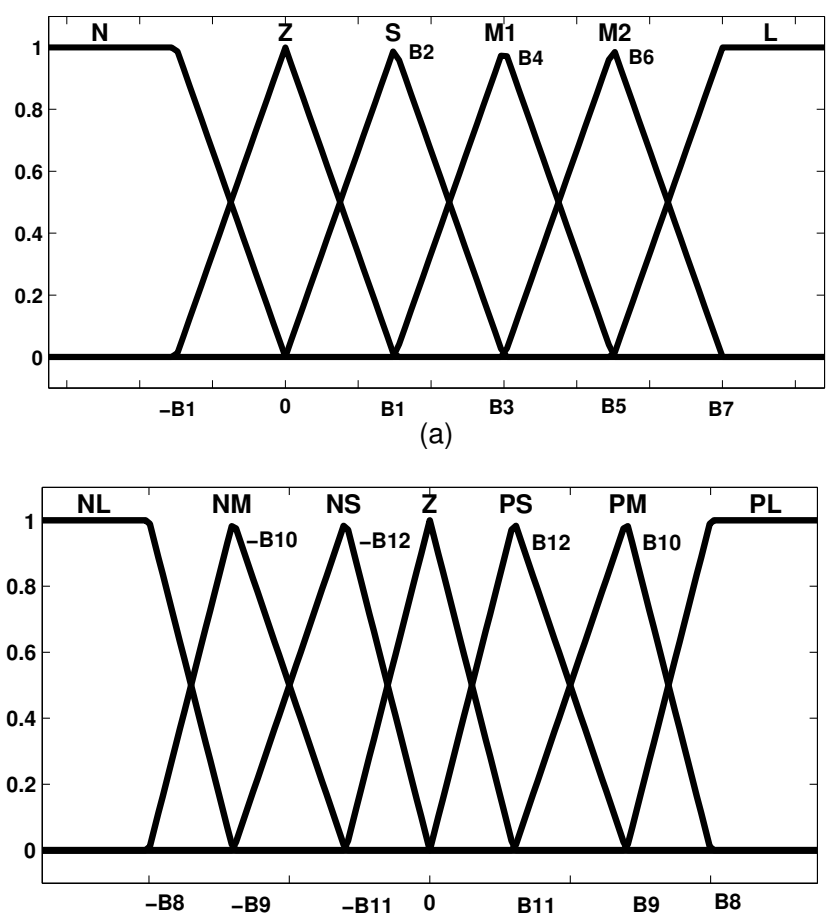

(b)

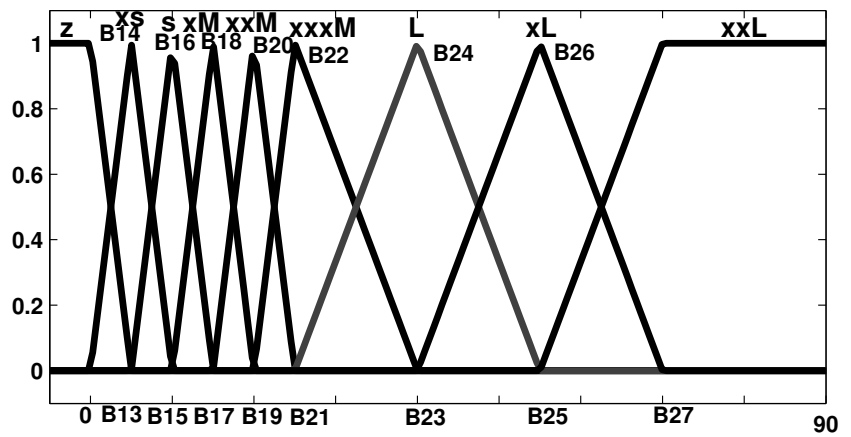

(c)

Figure 6. (a) Fuzzy membership functions for the pitch angle controller's first input. (b) Fuzzy membership functions for the pitch angle controller's second input. (c) Fuzzy membership functions for the pitch angle controller's output.

\subsection{Limitations of the Adopted Control Schemes}

The pitch angle control system presented in this paper works in all operating regions of the WTG. When the wind speed is from zero to the cut-in value, there is no power generated and the pitch angle is set as $\beta=90^{\circ}$. Meanwhile, when the wind speed is between the cut-in and rated values, respectively, the pitch angle is fixed at $\beta=0^{\circ}$ so that the power can be maximized. As the wind speed exceeds its rated value, the proposed pitch angle control system operates between $0^{\circ}$ and $90^{\circ}$ to maintain the rated 
power of the WTG. Above the cut-out speed, the pitch angle is again set as $\beta=90^{\circ}$ for safety reasons. $\beta$ is limited by a rate limiter $\left( \pm 20^{\circ} / \mathrm{s}\right)$ and a limiter within the range $\left(0^{\circ}-90^{\circ}\right)$. In addition, all power values are per unit (pu) on 100 MVA base. The maximum limit of active power in each transmission line is $200 \mathrm{MW}(2 \mathrm{pu})$.

The power system model has been linearized using commands of MATLAB ${ }^{\circledR}$ to check its stability performance including the proposed control schemes. The singular value plots of the control loops with/without the suggested controllers are shown in Figure 8. The frequency-closed control loop is specified with rotor speed command $\omega_{\text {ref }}$ as the input signal and $\omega$ as the output signal. The pitch angle closed control loop is formulated with $\Delta P_{W t g}$ as the input signal and $\beta_{C M D}$ is considered as the output signal. From this figure, it is seen that the singular value plot of the frequency control loop has an integral characteristic at the low-frequency domain without using the proposed control schemes. Because of this, the frequency deviations keep increasing so the characteristic can be eliminated. On the other hand, there is a high gain in the low-frequency domain (below $1 \mathrm{rad} / \mathrm{s}$ ) clarified in the pitch angle control loop without utilizing the intended control approaches. Therefore, the output power fluctuations of WTG will increase at the low-frequency domain.

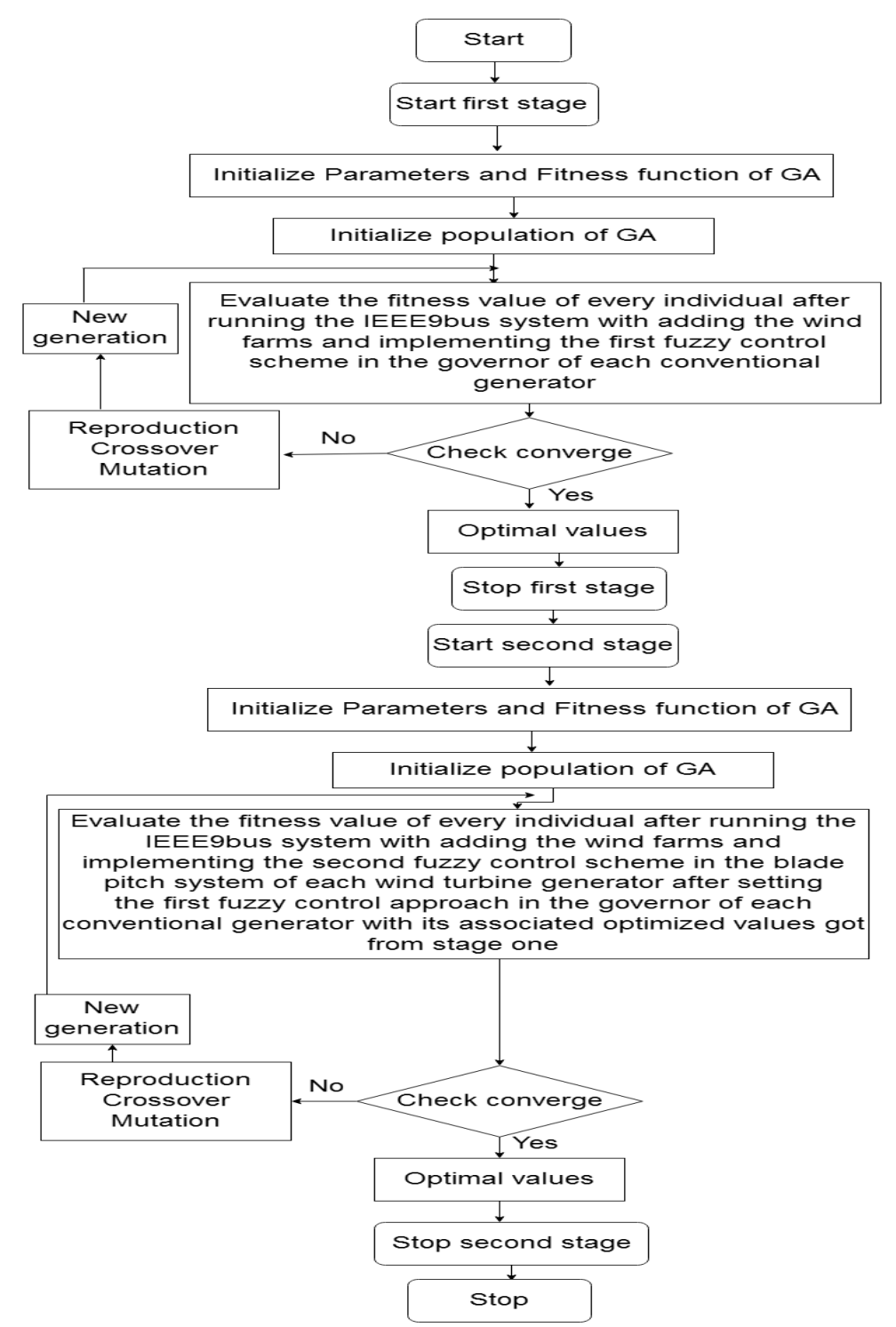

Figure 7. Flowchart of the design process of two GA-fuzzy-based control schemes. GA: genetic algorithm. 


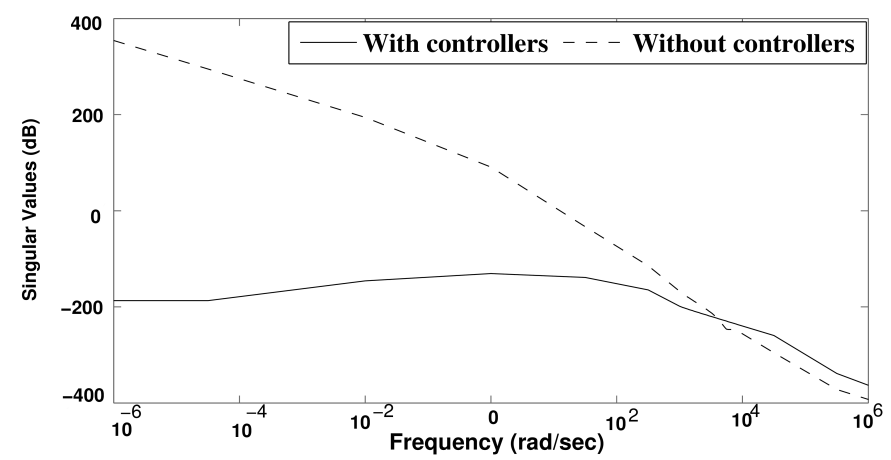

(a)

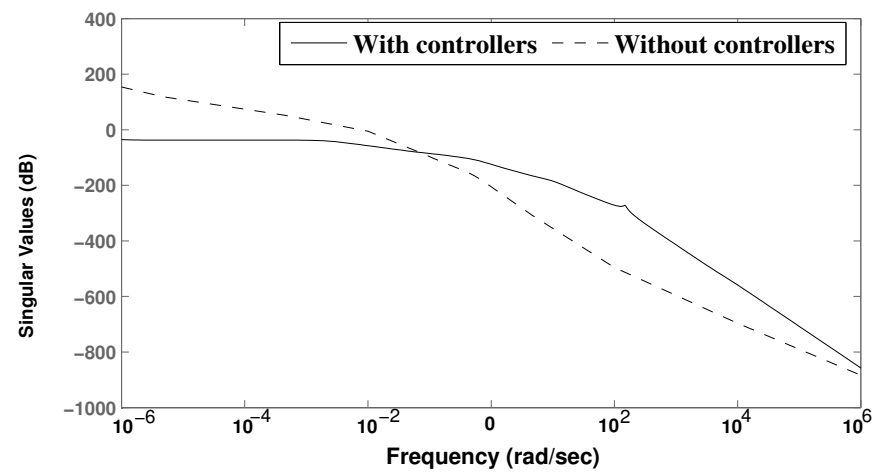

(b)

Figure 8. (a) Singular value plot of frequency control loop. (b) Singular value plot of the pitch angle control loop.

\section{Results}

To investigate the performance of the designed control schemes, the standard IEEE nine-bus three-generator system is considered as test model including three wind farms with $25 \%$ penetration. Simulation results show a comparison between the proposed methods and conventional ones. Actually, four cases are considered in simulation results. The first case represents the traditional methods consisting of the small power system without any controllers. The second and third cases are treated as the proposed cases in which the proposed governor controller for the conventional generators only, and both the designed governor and blade pitch controllers are implemented, respectively. The last case discusses the performance of the system under faulty operating conditions. Figure 9 shows the wind speed, which is modeled by random functions. The cut-in, rated, and cut-out wind speeds are considered as $5 \mathrm{~m} / \mathrm{s}, 12.5 \mathrm{~m} / \mathrm{s}$ and $25 \mathrm{~m} / \mathrm{s}$ respectively. The simulation is performed for $150 \mathrm{~s}$.

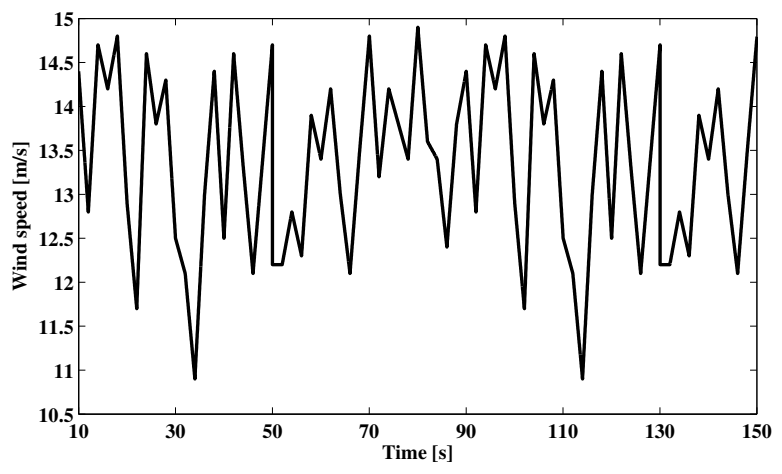

Figure 9. Wind speed. 


\subsection{Conventional Method (Case 1)}

No control approach is implemented for this case in the governor of the conventional generators or the pitch angle system of the WTG. Figure 10 shows the system frequency, generated output power of wind farms, and transmitted power in transmission lines, respectively. The results clearly indicate the instability of the power system represented in frequency oscillations. Also, there are significant fluctuations in the output power of each wind farm, which leads to transmitted power variation for all transmission lines.
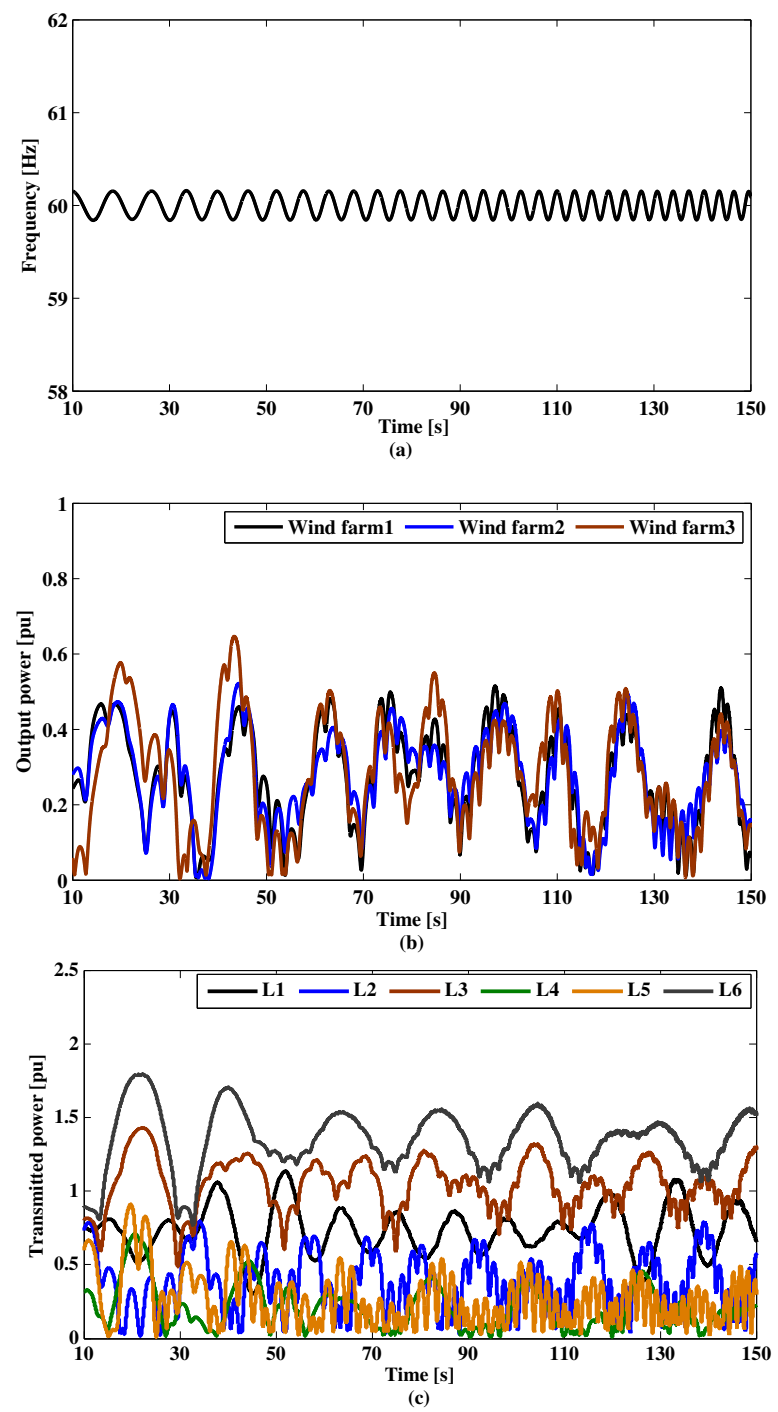

Figure 10. Case 1 result. (a) System frequency. (b) Generated output power of wind farms. (c) Transmitted power.

\subsection{Case 2}

The first designed GA-based fuzzy controller is implemented in the governor system of each conventional generator in this case to improve frequency stability of the power system. The obtained result in Figure 11 shows that the proposed controller can damp the frequency oscillations. On the other hand, due to the lack of the pitch angle controller in the WTG, fluctuations in the generated output power of wind farms still appear in the system, leading to variations in the transmitted power of transmission lines. 

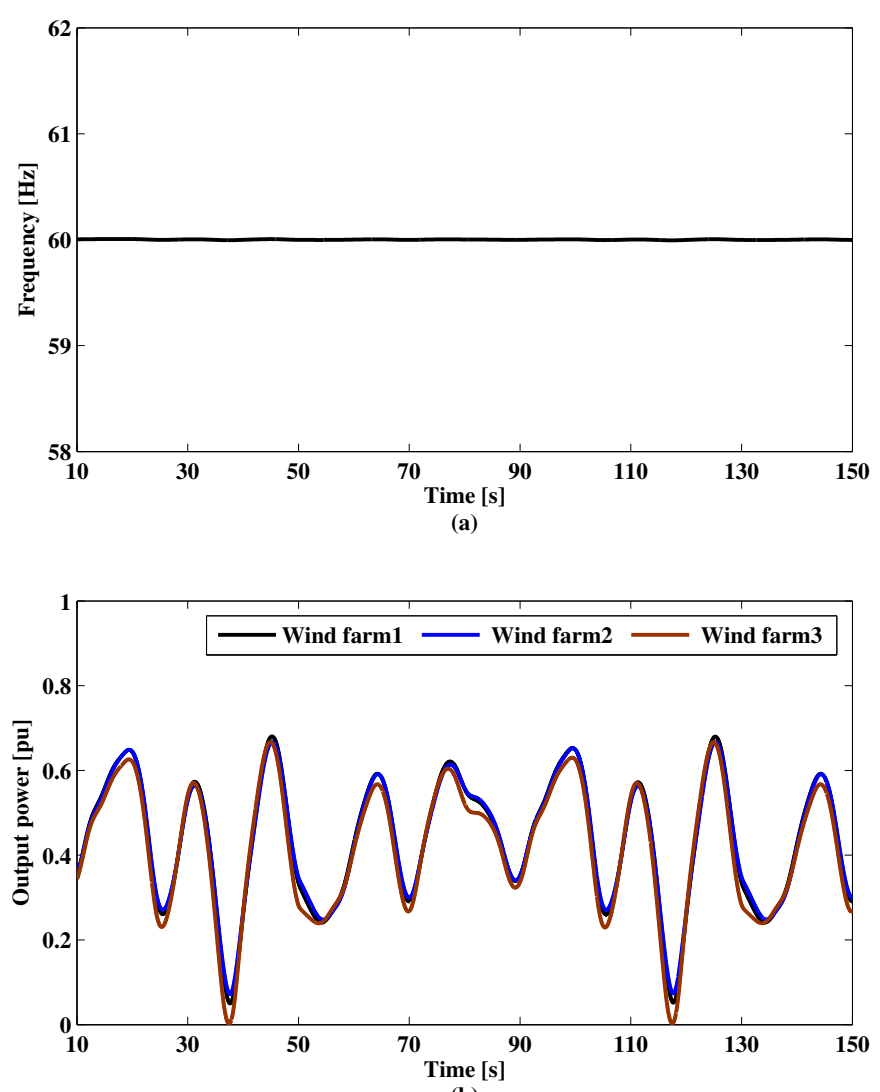

(b)

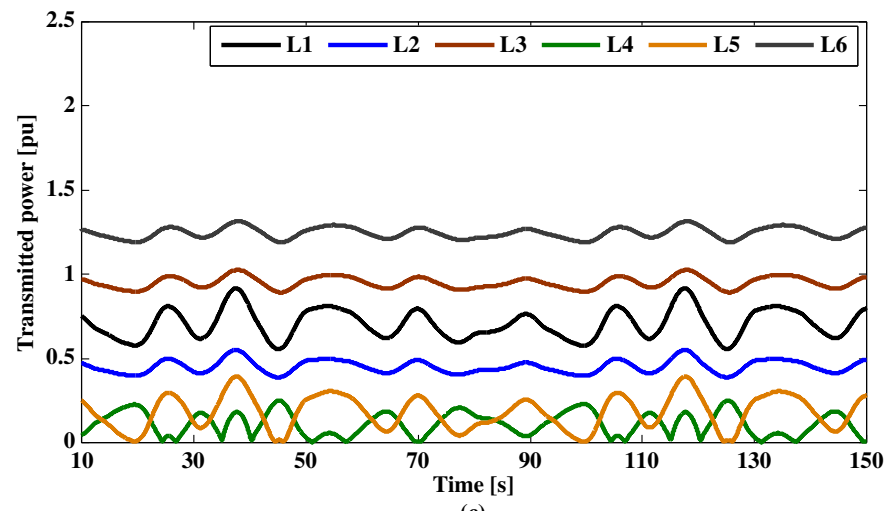

(c)

Figure 11. Case 2 result. (a) System frequency. (b) Generated output power of wind farms. (c) Transmitted power.

\subsection{Case 3}

The proposed two GA-based fuzzy controllers are implemented on their associated places in the power system for this case study. The first one is constructed in the governor system of each conventional generator, while the other one is used in the pitch angle control system of each WTG. The effectiveness of the proposed controllers in the test system is validated from the frequency and power points of view in Figure 12 that presents pitch angle of each wind turbine, system frequency, output power of wind farms, and transmitted power in transmission lines. Results confirm the ability of the proposed controllers to damp frequency oscillations and smooth the generated output power of wind farms to mitigate the transmitted power fluctuations in transmission lines. 

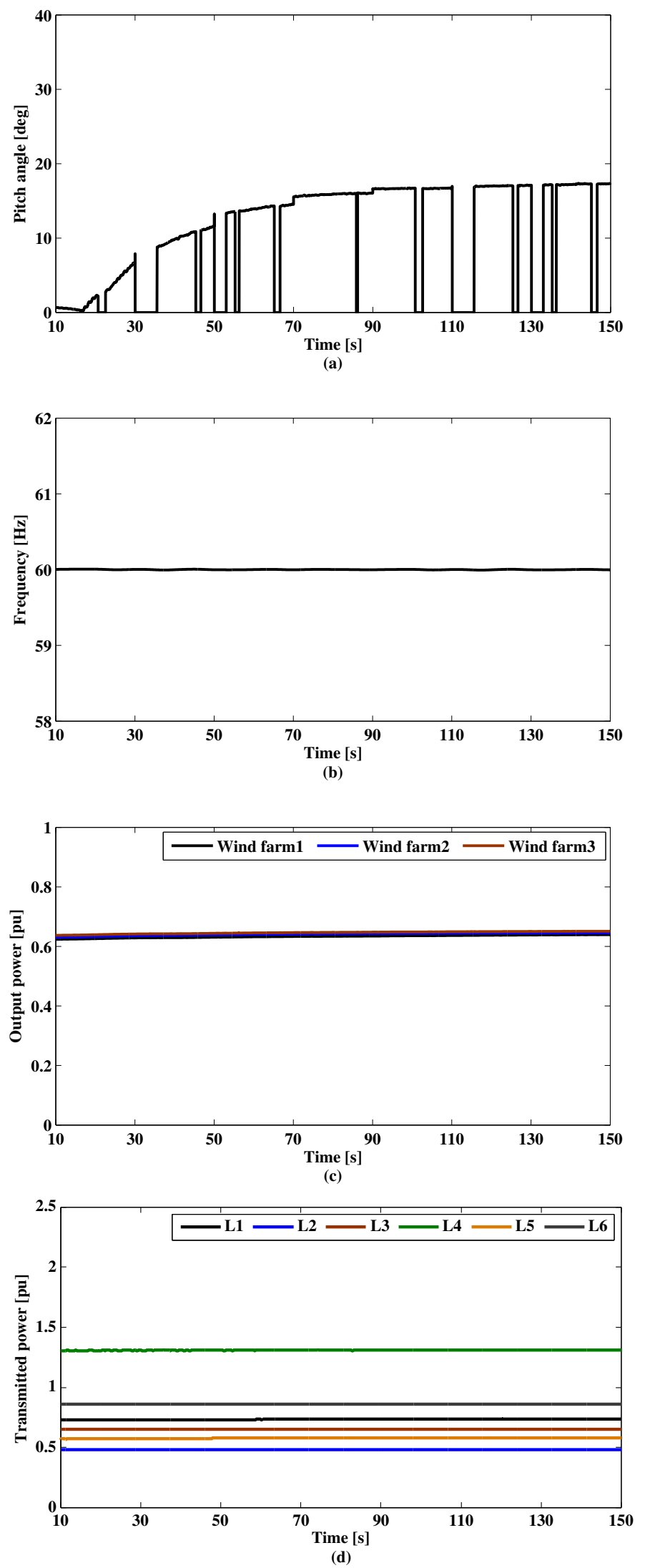

Figure 12. Case 3 result. (a) Pitch angle. (b) System frequency. (c) Generated output power of wind farms. (d) Transmitted power. 


\subsection{Faulty Operating Condition (Case 4)}

To confirm the robustness and effectiveness of the proposed controllers, performance of power system is studied under severe operating conditions represented as occurring three phases to the ground fault at the most loaded transmission line, which is line 4 from the previous case results. The fault occurs at time $t=30 \mathrm{~s}$ and is cleared at $t=50 \mathrm{~s}$. The associated results are indicated in Figure 13. Results confirm the ability of the system with the proposed controllers to withstand this harsh case study and guarantee good performance during the fault period. Also, the system can restore its normal operation after clearing the fault, which indicates its stability from the perspectives of frequency and power.

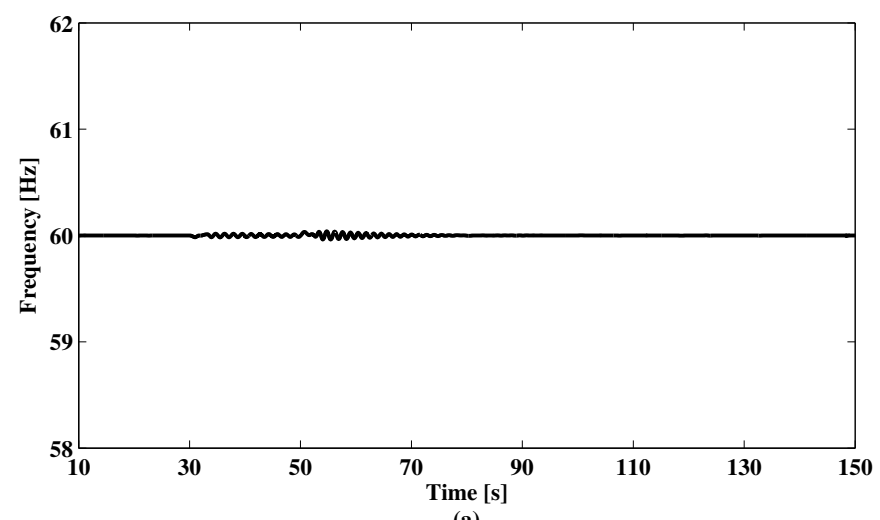

(a)
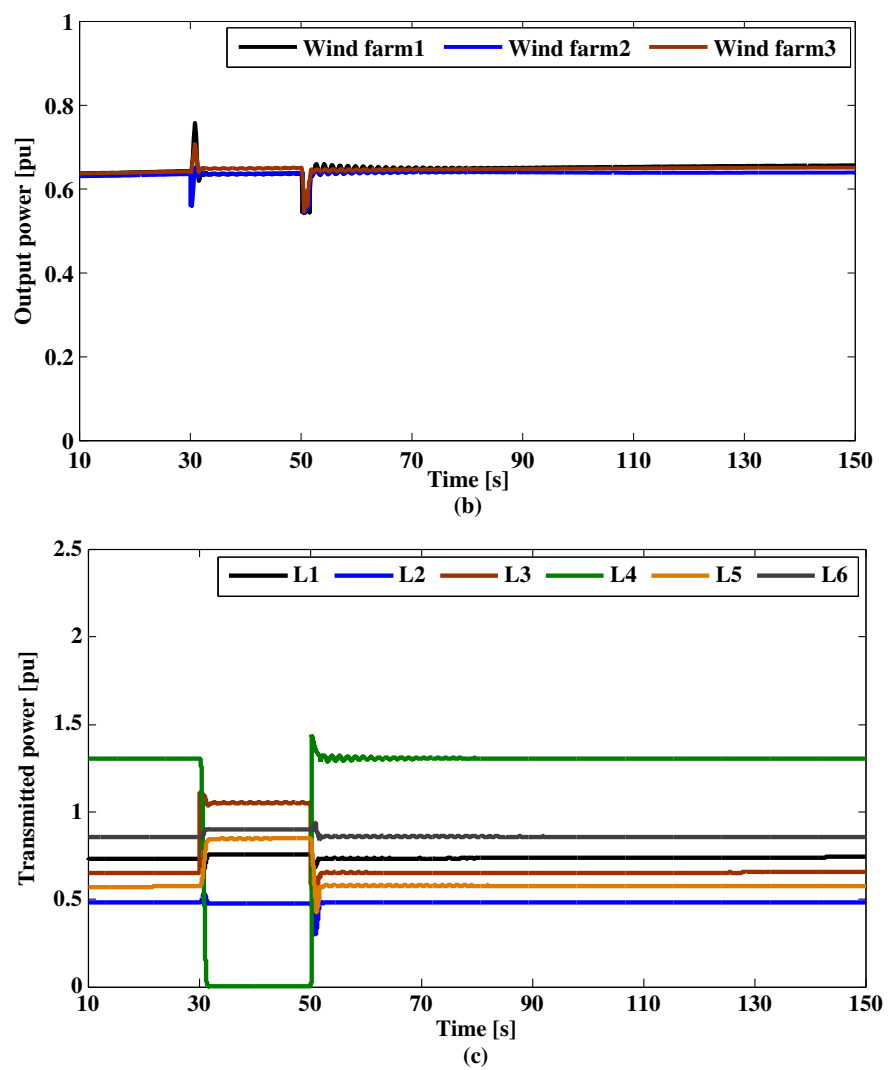

Figure 13. Case 4 result. (a) System frequency. (b) Generated output power of wind farms. (c) Transmitted power. 


\section{Result Analysis}

A detailed analysis of the time domain simulation results is presented here for the four case studies presented in the last section.

1. Case 1 represents the implementation of three wind farms in the stable IEEE nine-bus three-generator system with high penetration $(25 \%)$ that leads to significant system instability appearing in frequency oscillation around $60.2 \mathrm{~Hz}-59.8 \mathrm{~Hz}$. This forces frequency relays to trip in some portions of the system. Also, there are great fluctuations in the output power of wind farms in the range of 0-0.6 pu which leads to oscillations in the transmitted power between 0 and $1.7 \mathrm{pu}$. Hence, the majority of loads in this case cannot be met with a continuous power supply within acceptable frequency limits, which decreases the reliability and power quality of the system with harming loads, especially those are sensitive for frequency fluctuations and voltage flicker, with a decrease in their life time.

2. Case 2 shows the application of the first hybrid GA-fuzzy based control approach to the governor system of the conventional generators. As a result, the proposed constant frequency of $60 \mathrm{~Hz}$ is achieved as shown in Figure 11. Besides, output power fluctuations of wind farms still exist in range of 0-0.6 pu which derive to the transmitted power deviations in lower amounts compared to those in case 1 . Hence, the first controller succeeded in damping frequency oscillations but some fluctuations still appeared in power responses.

3. Case 3 involves the proposed scenario presenting implementation of the two hybrid GA-fuzzy based control approaches in the power system. The first approach is applied for the governor of conventional generators, while the second is used for the pitch angle control system of wind farms. Simulation results shown in Figure 12 confirm the ability of the first controller to keep frequency constant at $60 \mathrm{~Hz}$. Also, the second controller can suppress the output power fluctuations of wind farms and ensure constant power at around $0.63 \mathrm{pu}(63 \mathrm{MW})$ of each wind farm, even with variable wind speed, due to effective blade pitch angle control as shown in Figure 12. Beside this, control techniques succeeded to damp transmitted power oscillations ensuring continuous power supply to customers with high power quality.

4. To validate the robustness of the proposed control schemes in emergencies, a three-phase to ground fault at the most loaded transmission line is considered in case 4 . The fault occurs at $t=30 \mathrm{~s}$ and is cleared at $t=50 \mathrm{~s}$. Simulation results indicate the ability of the control techniques to damp frequency and wind power fluctuations, with reducing sudden overshoots at times of fault occurrence and clearance, respectively. As a result of transmission line outage, the transmitted power via the remaining transmission lines is redistributed to compensate the transmission line outage and ensure continuous power feeding for loads. After fault clearance, the power system comes back safely to its normal operating point thanks to the proposed control schemes with damping all power and frequency fluctuations and ensuring continuous power supply of the system with high power quality without any harmful effects to the sensitive loads even in severe operating conditions is ensured.

\section{Conclusions}

Two hybrid GA-fuzzy based control schemes are applied for a small power system in this paper. The power system consists of the standard IEEE nine-bus three-generator test model including three wind farms with $25 \%$ penetration of the overall system-rated capacity. Each wind farm is composed of 21 wind turbines with 3-MW rated capacity of each turbine. In the first scheme, the controller is constructed in the governor system of conventional generators to damp frequency oscillations. The second scheme is implemented for the pitch angle control system of wind turbines to smooth the output generated power fluctuations of wind farms and then improve the whole system performance. The GA is used to optimize the membership function parameters of fuzzy logic controllers to achieve optimal behavior. Four case studies are considered to investigate the impact of the proposed controllers 
for enhancing the performance of the power system from the frequency and power stability points of view under normal and faulty operating conditions. A case study with a fault is presented, which occurs as a three phase to ground fault at the most loaded transmission line. Simulation results show that the proposed controllers are able to damp frequency oscillations and also smooth the generated power of wind turbines, and consequently reduce the transmitted power fluctuations under normal operating conditions. Also, controllers can hold out in the faulty case and ensure adequate performance during this time, restoring the normal operation of the system after clearing the fault. Overall, the performance of the small power system is enhanced significantly using the proposed technique. The research can be considered as a complete study clarifying the effect of implementing high-penetration renewable energy sources in small power systems from the frequency and active power points of view, addressing the main defects of the previous studies that neglect the high nonlinear properties of wind turbines and their significant effect on the performance of the power system. Moreover, this research solves the main problem of FLC: complex and time-consuming procedures for defining the input and output membership function parameters. Implementing the practical model and comparing the obtained results with the outcomes of this research is a future mission, as we will extend the study to include voltage control strategies.

Author Contributions: All authors contributed to this work. Mohammed Elsayed Lotfy, Tomonobu Senjyu, and Mohammed Abdel-Fattah Farahat performed the research, discussed the results, and prepared the manuscript; Amal Farouq Abdel-Gawad, Liu Lei, and Manoj Datta suggested the research idea and contributed to writing and revising the paper. All authors revised and approved the manuscript.

Conflicts of Interest: The authors declare no conflict of interest.

\begin{tabular}{ll}
\multicolumn{2}{l}{ Abbreviations } \\
$L_{1}-L_{6}$ & Transmission lines of the IEEE nine-bus system \\
$W \cdot F_{1}-W . F_{3}$ & Three implemented wind farms in the IEEE nine-bus system \\
$\omega$ & Rotor speed \\
$R$ & Speed regulation of each generator \\
$\Delta f$ & Frequency deviation of power system \\
$\beta$ & Pitch angle \\
$\rho$ & Air density \\
$A$ & Rotor swept area \\
$r$ & Blade radius \\
$C_{p}$ & Aerodynamic power coefficient \\
$V_{w}$ & Wind speed \\
$\lambda$ & Tip speed ratio \\
$\omega_{w}$ & Blade rotational speed \\
$\beta_{C M D}$ & Pitch angle command \\
$\Delta P_{W t g}$ & WTG output power fluctuations
\end{tabular}

\section{References}

1. Hunter, R.; Eillot, G. Wind-Diesel Systems: A Guide to Technology and Its Implementation; Cambridge University Press: Cambridge, UK, 1994.

2. Nacfaire, H. Wind-Diesel and Autonomous Energy Systems; Elsevier Science Publishers Ltd.: Amsterdam, Netherlands, 1989. 
3. Dhanalakshmi, R.; Palaniswami, S. Application of multi stage fuzzy logic control for load frequency control of an isolated wind diesel hybrid power system. In Proceedings of the 2011 International Conference on Green Technology and Environmental Conservation (GTEC 2011), Chennai, India, 15-17 December 2011; pp. 309-315.

4. Chowdhury, M.; Hossienzadeh, N.; Shen, W. Smoothing wind power fluctuations by fuzzy logic pitch angle controller. Renew. Energy 2012, 38, 224-233.

5. Kamel, R.; Chaouachi, A.; Nagasaka, K. Wind power smoothing using fuzzy logic pitch controller and energy capacitor system for improvement Micro-Grid performance in islanding mode. Energy 2010, 35, 2119-2129.

6. Pahasa, J.; Ngamroo, I. Coordinated control of wind turbine blade pitch angle and PHEVs using MPCs for load frequency control of microgrid. IEEE Syst. J. 2016, 10, 97-105.

7. Supriyadi, C.; Nandar, A. Robust PI control of smart controllable load for frequency stabilization of microgrid power system. Renew. Energy 2013, 56, 16-23.

8. Ekelund, T. Speed control of wind turbines in the stall region. In Proceedings of the Third IEEE Conference on Control Applications, Glasgow, UK, 24-26 August 1994; pp. 227-232.

9. Shaked, U.; Soroka, E. On the stability robustness of the continuous time LQG optimal control. IEEE Trans. Autom. Control 1985, AC-30, 1039-1043.

10. Gampa, S.; Das, D. Real power and frequency control of a small isolated power system. Int. J. Electr. Power Energy Syst. 2015, 64, 221-232.

11. Viveiros, C.; Melicio, R.; Igreja, J.; Mendes, V. Performance assessment of a wind energy conversion system using a hierarchical controller structure. Energy Convers. Manag. 2015, 93, 40-48.

12. Lasheen, A.; Elshafei, A. Wind turbine collective-pitch control via a fuzzy predictive algorithm. Renew. Energy 2016, 87, 298-306.

13. Van, T.; Nguyen, T.; Lee, D. Advanced pitch angle control based on fuzzy logic for variable-speed wind turbine systems. IEEE Trans. Energy Convers. 2015, 30, 578-587.

14. Han, B.; Zhou, L.; Yang, F.; Xiang, Z. Individual pitch controller based on fuzzy logic control for wind turbine load mitigation. IET Renew. Power Gener. 2016, 10, 687-693.

15. Sakamoto, R.; Senjyu, T.; Urasaki, N.; Takagi, T.; Sugimoto, S. Output power leveling of wind turbine generator by pitch angle control using $H_{\infty}$ control. Electr. Eng. Jpn. 2008, 126, 17-24.

16. Corcuera, A.; Arrese, A.; Ezquerra, J.; Segurola, E.; Landaluze, J. $H_{\infty}$ based control for load mitigation in wind turbines. Energies 2012, 5, 938-967.

17. Muhando, B.; Wies, R. Nonlinear $H_{\infty}$ constrained feedback control for grid interactive WECS under high stochasticity. IEEE Trans. Energy Convers. 2011, 26, 1000-1009.

18. Bevrani, H.; Feizi, M.; Ataee, S. Robust frequency control in an islanded microgrid: $H_{\infty}$ and $\mu$-synthesis approaches. IEEE Trans. Smart Grid 2016, 7, 706-717.

19. Mohamed, T.; Morel, J.; Bevrani, H.; Hiyama, T. Model predictive based load frequency control design concerning wind turbines. Int. J. Electr. Power Energy Syst. 2012, 43, 858-867.

20. Tarkeshwar, M.; Mukherjee, V. Quai-oppositional harmony search algorithm and fuzzy logic controller for load frequency stabilization of an isolated hybrid power system. IET Gener. Transm. Distrib. 2015, 9, 427-444.

21. Lotfy, M.; Senjyu, T.; Farahat, M.; Abdel-Gawad, A.; Yona, A. A frequency control approach for hybrid power system using multi-objective optimization. Energies 2017, 10, 80.

22. Lotfy, M.; Senjyu, T.; Farahat, M.; Abdel-Gawad, A.; Yona, A. Enhancement of a small power system performance using multi-objective optimization. IEEE Access 2017, 5, 6212-6224.

23. Lotfy, M.; Senjyu, T.; Farahat, M.; Abdel-Gawad, A.; Matayoshi, H. A Polar fuzzy control scheme for hybrid power system using vehicle-to-grid technique. Energies 2017, 10, 1083.

24. Bevrani, H.; Daneshmand, P. Fuzzy logic-based load-frequency control concerning high penetration of wind turbines. IEEE Syst. J. 2012, 6, 173-180.

25. Bevrani, H.; Daneshmand, P.; Babahajyani, P.; Mitani, Y.; Hiyama, T. Intelligent LFC concerning high penetration of wind power: synthesis and real-time application. IEEE Trans. Sustain. Energy 2013, 5, 655-662.

26. Bevrani, H.; Ghosh, A.; Ledwich, G. Renewable energy sources and frequency regulation: survey and new perspectives. IET Renew. Power Gener. 2010, 4, 438-457.

27. Li, Y.; Tong, S.; Li, T. Hybrid fuzzy adaptive output feedback control design for uncertain MIMO nonlinear systems with time-varying delays and input saturation. IEEE Trans. Fuzzy Syst. 2016, 24, 841-853. 
28. Li, Y.; Tong, S.; Li, T. Observer-based adaptive fuzzy tracking control of MIMO stochastic nonlinear systems with unknown control directions and unknown dead-zones. IEEE Trans. Fuzzy Syst. 2015, 23, 1228-1241.

29. Li, Y.; Tong, S.; Li, T. Composite adaptive fuzzy output feedback control design for uncertain nonlinear strict-feedback systems with input saturation. IEEE Trans. Cybern. 2015, 45, 2299-2308.

30. Li, H.; Wu, C.; Shi, P.; Gao, Y. Control of nonlinear networked systems with packet dropouts: interval type-2 fuzzy model-based approach. IEEE Trans. Cybern. 2015, 45, 2378-2389.

31. Wang, T.; Zhang, Y.; Qiu, J.; Gao, H. Adaptive fuzzy backstepping control for a class of nonlinear systems with sampled and delayed measurements. IEEE Trans. Fuzzy Syst. 2015, 23, 302-312.

32. Sawle, Y.; Gupta, S.; Bohre, A. PV-wind hybrid system: A review with case study. Cogent Eng. 2016, 3, 1189305.

33. Ou, T.; Hong, C. Dynamic operation and control of microgrid hybrid power systems. Energy 2014, 66, 314-323.

34. Hong, C.; Ou, T.; Lu, K. Development of intelligent MPPT (maximum power point tracking) control for a grid-connected hybrid power generation system. Energy 2013, 50, 270-279.

35. Shiau, J.; Wei, Y.; Lee, M. Fuzzy controller for a voltage-regulated solar-powered MPPT system for hybrid power system applications. Energies 2015, 8, 3292-3312.

36. Ou, T.; Su, W.; Liu, X.; Huang, S.; Tai, T. A modified bird-mating optimization with hill-climbing for connection decisions of transformers. Energies 2016, 9, 671.

37. Ou, T. A novel unsymmetrical faults analysis for microgrid distribution systems. Int. J. Electr. Power Energy Syst. 2012, 43, 1017-1024.

38. Ou, T. Ground fault current analysis with a direct building algorithm for microgrid distribution. Int. J. Electr. Power Energy Syst. 2013, 53, 867-875.

39. Gonzalez, A.; Riba, J.; Ruis, A.; Puig, R. Optimal sizing of a hybrid grid-connected photovoltaic and wind power system. Appl. Energy 2015, 154, 752-762.

40. Ou, T.; Lu, K.; Huang, C. Improvement of transient stability in a hybrid power multi-system using a designed NIDC (novel intelligent damping controller). Energies 2017, 10, 488.

41. Debnath, M.; Jena, T.; Mallick, R. Optimal design of PD-fuzzy-PID cascaded controller for automatic generation control. Cogent Eng. 2017, 4, 1416535.

42. Upadhyay, S.; Sharma, M. A review on configurations, control and sizing methodologies of hybrid energy systems. Renew. Sustain. Energy Rev. 2014, 38, 47-63.

43. Anderson, P.; Fouad, A. Power System Control and Stability; IEEE Press: New York, NY, USA, 1994.

44. Sudha, K.; Santhi, R. load frequency control of an interconnected reheat thermal system using Type-2 fuzzy system including SMES units. Int. J. Electr. Power Energy Syst. 2012, 43, 1383-1392.

45. Simoes, M.; Bose, B.; Spiegel, R. Design and performance evaluation of fuzzy-logic-based variable-speed wind generation system. IEEE Trans. Ind. Appl. 1997, 33, 956-965.

46. Ross, T. Fuzzy Logic with Engineering Applications; John Wily \& Sons Ltd.: Hoboken, NJ, USA, 2004.

47. Tsoukalas, L.; Uhrig, R. Fuzzy and Natural Approaches in Engineering; Wiley: New York, NY, USA, 1997.

48. Goldberg, D. Genetic Algorithm in Search, Optimization and Machine Learning; Addison-Wesley Publishing Company Inc.: Boston, MA, USA, 1989.

49. Shivakumar, R.; Lakshmipathi, R. Implementation of an innovative bio inspired GA and PSO algorithm for controller design considering steam GT dynamics. Int. J. Comput. Sci. Issues 2010, 7, 18-28.

50. Pand, S.; Yadav, J.; Patidar, N.; Ardil, C. Evolutionary Techniques for model order reduction of large scale linear systems. Int. J. Eng. App. Sci. 2009, 5, 22-28.

51. Lee, D.; Wang, L. Small-signal stability analysis of an autonomous hybrid renewable energy power generation/energy storage system part I: time-domain simulations. IEEE Trans. Energy Convers. 2008, $23,311-320$.

(C) 2018 by the authors. Licensee MDPI, Basel, Switzerland. This article is an open access article distributed under the terms and conditions of the Creative Commons Attribution (CC BY) license (http:/ / creativecommons.org/licenses/by/4.0/). 